\title{
New insights into the mechanisms of organ-specific breast cancer metastasis
}

\author{
Girieca Lorusso $^{\mathrm{a}, \mathrm{b}, *}$, Curzio Rüegga,b,* \\ a Chair of Pathology, Department of Medicine, Faculty of Science, University of Fribourg, Fribourg, Switzerland \\ ${ }^{\mathrm{b}}$ National Center for Competence in Research (NCCR) Molecular Oncology, Swiss Institute of Experimental Cancer Research, Ecole Polytechnique Fédérale de Lausanne (ISREC-EPFL), \\ Lausanne, Switzerland
}

\begin{abstract}
Despite the substantial advances obtained in the treatment of localized malignancies, metastatic disease still lacks effective treatment and remains the primary cause of cancer mortality, including in breast cancer. Thus, in order to improve the survival of cancer patients it is necessary to effectively improve prevention or treatment of metastasis. To achieve this goal, complementary strategies can be envisaged: the first one is the eradication of established metastases by adding novel modalities to current treatments, such as immunotherapy or targeted therapies. A second one is to prevent tumor cell dissemination to secondary organs by targeting specific steps governing the metastatic cascade and organ-specific tropism. A third one is to block the colonization of secondary organs and subsequent cancer cell growth by impinging on the ability of disseminated cancer cells to adapt to the novel microenvironment. To obtain optimal results it might be necessary to combine these strategies. The development of therapeutic approaches aimed at preventing dissemination and organ colonization requires a deeper understanding of the specific genetic events occurring in cancer cells and of the host responses that co-operate to promote metastasis formation. Recent developments in the field disclosed novel mechanisms of metastasis. In particular the crosstalk between disseminated cancer cells and the host microenvironment is emerging as a critical determinant of metastasis. The identification of tissue-specific signals involved in metastatic progression will open the way to new therapeutic strategies. Here, we will review recent progress in the field, with particular emphasis on the mechanisms of organ specific dissemination and colonization of breast cancer.
\end{abstract}

\section{Introduction}

Despite recent major advances in the understanding of the mechanisms of breast cancer progression and in the development of novel therapeutic modalities, breast cancer remains the second leading cause of mortality among women. Mortality is almost invariably due to metastasis. For example, between 25\% and 50\% of patients diagnosed with breast cancer will eventually develop deadly metastases, often decades after the time of diagnosis and removal of the primary tumor. The prognosis for patients with metastatic breast cancer is generally unfavorable, with an average 5 -year survival rate of only about $25 \%[1,2]$. The different histological subtypes of breast cancer (e.g. ductal, lobular, basallike) and molecular marker expression (e.g. estrogen receptor, ER; progesterone receptor, PR; human epidermal growth factor

\footnotetext{
* Corresponding authors at: Chair of Pathology, Department of Medicine, Faculty of Science, University of Fribourg, 1 Route Albert Gockel, CH-1700 Fribourg, Switzerland. Tel.: +41 21300 8766; fax: +41213009733.

E-mail addresses: girieca.lorusso@unifr.ch (G. Lorusso), curzio.ruegg@unifr.ch (C. Rüegg).
}

receptor 2, HER2) have strong prognostic and predictive values. For example, triple negative breast cancers (i.e. ER ${ }^{-}, \mathrm{PR}^{-}, \mathrm{HER} 2^{-}$ negative) are associated with a significantly increased risk of progression and metastasis formation. In spite of intense clinical research efforts, only limited advances have been obtained in the management of breast cancer metastases. Therapeutic options are mainly based on the systemic administration of cytotoxic chemotherapy, or radiotherapy and surgery for isolated lesions, but the long-term impact on survival is limited [3]. Metastatic disease still remains the most critical condition limiting patient survival, and the development of effective treatment against metastatic cancers, including breast cancer, is among the most outstanding challenge in current experimental and clinical cancer research. Although surgery, chemotherapy and radiation therapy can control primary tumor growth, they are not very effective in preventing relapses. It has been recently demonstrated that targeted immunotherapy against tumor tissue can generate an adaptive immune response mediated by cytotoxic $T$ cells that further targets distant metastatic sites [4]. The process of metastasis has been traditionally viewed as a multi-step sequential cascade of events starting from local invasion at site of primary tumor, progression toward hematogenic or lymphogenic spreading, colonization 
and outgrowth at distant sites [5]. Metastases are themselves often source of further dissemination to additional tissues. Recent experimental evidence revealed that cancer cells recirculate from metastatic lesions back to the primary tumor [6,7]. This observation, if confirmed in human cancer, would have important implications to prediction and therapy.

One striking feature of the metastatic spread is organ-specificity. Recent discoveries indicate that both molecular characteristics of the cancer cells and of the target tissue cooperate to determine the organotropism of metastasis observed for many tumors [8,9]. The dissection of the mechanisms by which different tumor types spread to specific organs and the identification of the molecules and pathways involved is one of the emerging topic of investigation in current cancer research [10]. Unraveling these molecules and pathways might open the way for unprecedented therapeutic opportunities to translate into the clinic. Here we will review some of the emerging concepts and recent findings in breast cancer metastasis, and discuss their biological and clinical relevance as well as some of the remaining and emerging open questions.

\section{Spatial-temporal models of tumor cell dissemination}

To develop clinically detectable metastases, cancer cells must complete chronologically and functionally well-defined and interrelated steps, globally referred to as the metastatic cascade. Tumor cells need first to migrate out of the primary tumor, enter into the bloodstream (or lymphatic vessels) and to seed at distant, secondary sites $[11,12]$. The disseminated tumor cells then need to evade the host immune responses and to adapt to the local "foreign" microenvironment in order to survive, proliferate and successfully form secondary tumors [13]. The basic events of the metastatic cascade, such as initial invasion and circulation in the blood are shared by different tumor types, while other ones, in particular the colonization of specific organs and secondary growth, are specific for distinct tumor types and different target organs [8-10]. The metastatic process itself is believed to be highly inefficient, since only a small fraction of cancer cells entering the circulation will successfully generate secondary tumors. This low metastatic efficiency is considered to be the result of tumor cell death in the blood circulation, caused by the exposure to shear stress and hemodynamic forces and induction of anoikis due to lack of adhesion to matrix and to immune attack by cytotoxic effector cells [14,15].

Traditionally, it is considered that metastatic dissemination occurs late during tumor progression, at a time when the primary tumor has already reached a considerable volume [16] (Fig. 1, upper panel). Indeed, for most cancers, the primary tumor volume represents a risk factor for progression to metastasis: the greater the volume, the higher the risk. In this model, also called the linear model of metastasis, genetic modifications progressively accumulate in cancer cells, whereby cells with advantageous mutations will survive and expand in the primary tumor (clonal evolution theory of cancer). Based on this model, cells acquiring metastatic capacities will initially form a small cell subpopulation within the primary tumor. Accordingly, metastasis-driving mutations should be rare in the primary tumor but frequent in the distant metastases $[2,12]$. The observation that metastases and their corresponding primary tumor have similar molecular signatures has been interpreted as evidence for this model [17-19].

Recently, however, this linear model of metastasis has been challenged by observations suggesting that cancer cells, in particular in breast cancer, might disseminate early during tumor progression, i.e. at a stage when the primary lesion is small or possibly even at pre-malignant stages (parallel model of metastasis) (Fig. 1, lower panel). This model implies that disseminated cells evolve independently of the primary tumor [20]. In
Linear model

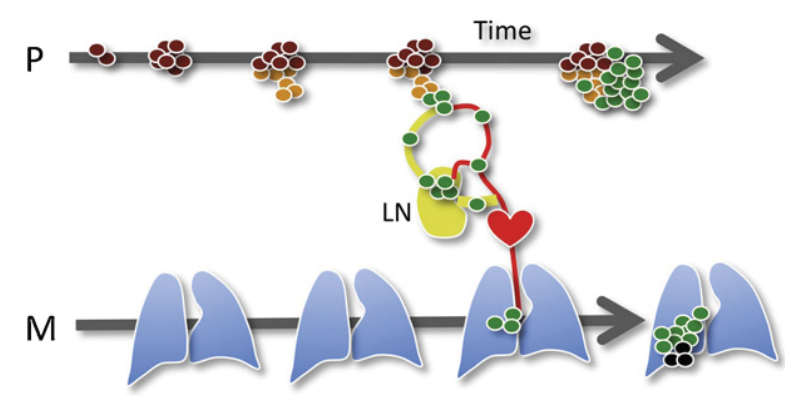

Parallel model

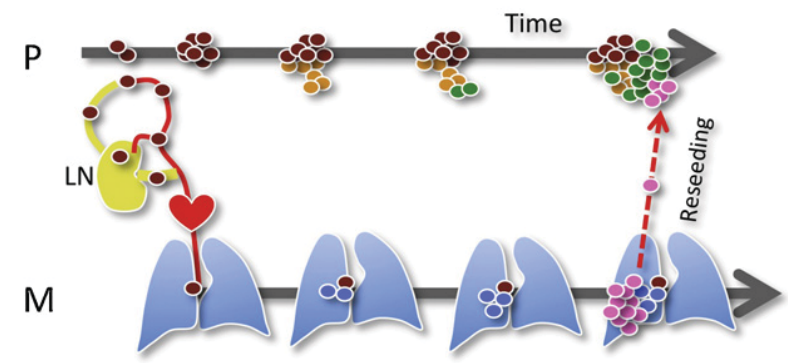

Fig. 1. Linear and parallel models of metastasis. In the linear model of metastasis (upper panel), genetic modifications progressively accumulate in cancer cells of the primary tumor, whereby cells with advantageous mutations will survive and expand through clonal evolution. Cells acquiring metastatic capacities will form a small cell subpopulation within the primary tumor which, when disseminated, are more effective in colonizing distant organs. In the parallel model of metastasis (lower panel), it is proposed that cancer cells disseminate early during tumor progression, at a stage when the primary lesion is small or possibly even pre-malignant. Disseminated cells then evolve independently of the primary tumor to form metastases. In breast cancer both models are supported by clinical and experimental evidences. Metastatic cells can disseminate through lymphatic vessels and lymph nodes first or directly through the blood stream. Cancer cells recirculating from metastases to primary tumors might contribute to bad prognostic signatures. P, primary tumor; M, metastasis. See Refs. [20,21,24,26].

support of this model there are observations demonstrating that genetic alterations occurring in breast cancer cells disseminated into the bone marrow often do not resemble to those present in the corresponding primary tumor $[21,22]$. Based on this model, the genetic alterations in disseminated tumor cells should be more relevant in predicting response to therapy in metastatic disease than those found in the primary tumor [23]. Tumor cells circulating in the peripheral blood or disseminated tumor cells in the bone marrow can be characterized at the single-cell level and may serve as a powerful tool for detection of early metastatic spreading and determining the suitable therapy [24].

The debate on the linear versus parallel models of metastasis raises three fundamental questions about the origin of metastatic cancer cells, for which today we have no definitive answer: when, where and how do metastatic cells arise in the course of a cancer disease? This capability may arise very early within the primary tumor even prior to invasion and dissemination, or it may be acquired very late among the already disseminated cancer cells at a time when they need to adapt, survive and growth in the newly colonized tissue environment $[25,26]$.

\section{Breaking the wall}

The first obstacle encountered by circulating tumor cells to enter distant organs is the vascular wall, in particular the endothelial 
lining. In some organs, like the bone marrow or the liver, the microvessels are constituted by highly permeable sinusoids. Sinusoids are permissive for the physiological extravasation of blood cells and represent weak barriers to cancer cells. In contrast, in most other organs endothelial cells form a contiguous lining through which cancer cells cannot freely penetrate. An organ with an extremely tight endothelium is the brain with its peculiar blood-brain barrier. Metastasis is facilitated by blood platelets and leukocytes, which form complexes by L- or P-selectins with tumor cells, thereby contributing to the arrest of such emboli in the vasculature [27]. Enhanced expression of selectin ligands (sLex/a, sialyl Lewis x/a glycans) is associated with metastatic progression, and correlates with poor prognosis for patients. The extravasation of circulating tumor cells through tight vascular beds requires the expression of genes that will remodel the vasculature to increase permeability $[10,13,28]$. Stroma-derived TGF- $\beta$ enhances the metastatic tropism of breast cancer cells to lung by inducing ANGPTL4 gene expression, which remodels the vasculature and facilitates tumor cell extravasation [29]. Specific organ tropism also involves the networking of cytokines and chemokines expressed in the target tissue and interacting with their cognate receptors expressed on tumor cells. For example, the CXCR4 and CCR7 receptors and their ligands CXCL12 and CCL21 respectively, are specifically used by breast cancer cells to arrest and migrate into secondary organs [30,31].

\section{Adapting to the new environment}

The second obstacle for a disseminated cancer cells to form growing metastases is their ability to adapt to the foreign tissue. Physiologically, normal cells survive and proliferate within their specific original tissue, while they are unable to do so outside of it [32]. This is considered as a critical mechanism to maintain correct tissue identity and tropism [8,33]. In contrast, disseminated cancer cells can grow in organs and tissues often very different from their origin. For this cancer cells must acquire novel capacities, in particular the ability to interact with the extracellular matrix and with cells of the new microenvironment. Tumor cells establish a bidirectional relationship with the surrounding stroma very early during tumor progression, namely at the time of initial invasion. Thereafter, tumor-stroma interactions actively contribute to tumor progression toward metastasis. Tumor cells themselves can co-opt the colonized tissue stroma and impinge on many physiological events favoring tumor growth and progression, such as twisting and polarizing inflammation, suppressing the immune response, promoting angiogenesis, or releasing growth, survival and motility factors [25]. In response to tumor cells, resident stromal cells can profoundly alter their transcriptome to express genes providing a favorable environment for the disseminated cancer cells [34,35].

\subsection{The pre-metastatic niche}

Since tissue modifications have been observed prior to tumor cell seeding, the concept of pre-metastatic niche has been introduced. The building of an adequate pre-metastatic niche is key for the initial survival of the tumor cells disseminated in a noncompatible organ $[36,37]$. Kaplan et al. highlighted the role of mobilized bone marrow-derived cells (BMDCs) in creating a suitable microenvironment for the metastatic colonization of the lung. BMDCs are mobilized by factors released by the primary tumor and are directed to the prospective sites of metastasis prior to the arrival of the disseminated tumor cells [38,39]. These cells express VEGFR1, CD133, CD34, and c-Kit, all markers characteristic of hematopoietic progenitor cells (HPCs). Recruited VEGFR $1^{+}$cells can determine the metastatic pattern characteristic of different tumor cell types through the secretion of specific factors. Several molecular mechanisms involved in the formation of the premetastatic niche have been reported. The expression of VLA-4 ( $\alpha 4 \beta 1$ integrin) by VEGFR $1^{+}$BMDCs is essential for their interaction with fibronectin deposited in the pre-metastatic niche [38]. Once recruited, BMDCs are stimulated to secrete matrix metalloproteinases (MMPs), which process the matrix and release matrix-bound factors such as VEGF.

\subsection{Molecules promoting the pre-metastatic niche}

VEGFR1 signaling in BMDC and stromal cells, is essential for the activation of tumor-recruited BMDCs, for promoting vascular permeability facilitating tumor cell trans-endothelial migration, tumor cell survival as well as epithelial-to-mesenchymal transition (EMT) [40]. Hiratsuka et al. showed that VEGFR1 signaling is required for the induction of MMP-9 by endothelial cells and macrophages in the pre-metastatic lung [41]. The inflammatory chemoattractants S100A8 and S100A9 not only act in the primary tumor microenvironment, but they also contribute to the recruitment of $\mathrm{CD} 11 \mathrm{~b}^{+}$ myeloid cells to the pre-metastatic lung and to promote tumor cell survival through toll-like receptor TLR-4-dependent NF- $\mathrm{B}$ signaling $[42,43]$. Erler et al. described lysyl oxidase (LOX) as a critical molecule in the formation of the pre-metastatic niche. LOX co-localizes with fibronectin in lung basement membrane and crosslinks collagen IV to generate a suitable extracellular matrix that facilitates the recruitment of $\mathrm{CD} 11 \mathrm{~b}^{+}$myeloid cells and tumor cell survival [44-46]. The importance of the extracellular matrix in metastasis formation is further supported by the observation that in breast cancer tumor cells infiltrating the lung need to induce stromal osteopontin expression in order to initiate colonization [46]. Periostin was recently reported to promote maintenance of disseminated breast cancer stem cells in the lung through the recruitment of Wnt ligands and induction of Wnt signaling [47]. The extracellular matrix protein tenascin- $C$, which is expressed in normal stem cell niches is also expressed by aggressive breast cancer cells forming pulmonary metastasis to promote the survival and outgrowth of micrometastases [48]. Chemokines are further key players in metastasis formation. For example CXCL12 (also known as SDF1, stromal-derived factor 1) favors homing to target organs, in particular the bone marrow, of circulating tumor cells expressing the cognate receptor CXCR4. The CXCL12/CXCR4 axis creates a pre-metastatic niche in the bone for osteotropic cancers most notably mammary, prostatic, and ovarian cancers [30,31]. Similarly, CCL12/CCR7 and CCL22/CCR4 axes have been described to promote the dissemination of breast and lung cancer cells, respectively. Tumor cells expressing these chemokine receptors find a fertile soil within the foreign bone microenvironment due to the secretion of their specific chemokine ligands by osteoblasts [30]. Further, osteoblasts expressing angiopoietin (Ang-1) and osteopontin, are able to attract and recruit tumor cells to bone. Additional studies are required to characterize other molecular mechanisms involved in the pre-metastatic niche formation during the colonization and homing, in particular to other relevant target organs, such as brain or liver [39,49].

\section{Organotropism of metastasis}

Another striking feature of metastasis is that their localization does not occur randomly but rather at selected preferred sites. The blood circulation pattern provides only a partial explanation for the organ-specific colonization by metastatic cancers observed in patients $[28,50]$. For example, breast cancer often metastasizes to lung, bone, liver and brain, all sites that have no immediate direct vascular connections with the mammary gland tissue. 
Rather, metastatic outgrowth requires a proper microenvironment where cancer cells can survive, initiate proliferation and establish a new tumor mass [9]. This notion is not new, since Stephen Paget proposed it already in 1889 as the "seed and soil" hypothesis. He postulated that a successful colonization of a secondary organ depends on the intrinsic properties of the tumor cell itself ("seed") and a permissive and supportive role of the environment ("soil"), which co-operate to allow for survival and proliferation [13,51]. The first experimental evidence underneath the notion that cancer do not spread randomly was provided much later by Isaiah Fidler in 1973, who showed that different subpopulations of cancer cells isolated from specimens of the same cancer patient and injected into mice upon the same route, displayed distinctive patterns of organ colonization [52].

Four decades of subsequent studies, revealed several molecular aspects governing metastatic tropism and identifying mediators of site-specific outgrowth. In particular, genes whose expression specifically mediates metastatic colonization of breast cancer to bone, lung and brain have been identified [53-55]. These genes orchestrate the organ-specific pattern of metastatic spreading by overcoming the incompatibilities between the intrinsic features of cancer cells and the "obstacles" imposed by the foreign microenvironment. The metastatic tropism implies distinct adaptive programs determined by the organ where the metastatic colonization and outgrowth occurs, as well as by the tissue of origin of the metastatic cells. This double-faceted feature of the metastatic tropism may explain why breast carcinoma cells metastasizing to different organs, such as lung, bone, liver or brain, develop distinct genetic programs for each metastatic site. However, organ-specific mechanisms of metastasis are not universal. Different cancers, such as breast, prostate or lung, can metastasize to the same target organ by developing different molecular programs and activate distinct signaling pathways. The pleiotropic mechanisms of metastatic colonization can be interpreted as the result of the pattern of accumulated genetic modifications in the cancer cells necessary for primary tumor growth and dissemination and the complementary modifications required for successful survival and growth in the foreign environment. Functions already acquired during primary tumor growth will not need to be acquired again during metastasis and might therefore escape detection as pro-metastatic genes, although functionally they are (see Section 6). This multiplicity is further complicated by different temporal patterns of metastasis. For example, some carcinoma such as lung or pancreas, can form metastases in a relatively short period of time, whereas other carcinoma, such as breast, often take years to give rise to metastases. This suggests that some cancer cell types, such as pancreas, can rapidly embrace programs to adapt to the foreign microenvironment, while other cancer cell types, such as breast, require more time to activate programs for accomplishing the same result $[34,35,56]$. Considering the genetic instability of cancer cells, and the fact that only a small sub-population is able to overcome the multiple barriers on the path to metastasis, it is likely that at each step of the process some crucial genes are modified either through mutational events (i.e. amplification, translocation or loss of heterozygosity), or through transcriptional regulation, including epigenetic modifications $[10,35]$.

\section{Metastasis-promoting genes}

In order to identify molecular mediators of organ-specific metastasis, gene expression profiling experiments on cancer cells with different tropism in experimental models and clinical samples were performed. Globally, the obtained results indicate that breast carcinoma cells acquire genetic programs and specific patterns enabling their subsequent preferential colonization to specific target organs already within the primary tumor and that only a few additional events are required for full metastatic capacity. Functional experiments validated a number of critical genes mediating organ-specific metastases in breast cancer [57,58]. This approach also proved successful in classifying genes based on their general contribution to metastasis. Three principal categories have been proposed: genes promoting initiation, progression or virulence. Metastasis initiation genes comprise genes providing an advantage in tumor cell growth, escape and invasiveness at the primary tumor site, thereby facilitating intravasation into capillaries and hematic dissemination to distant organs. Metastasis virulence genes provide survival advantages to disseminated tumor cells within the newly colonized microenvironment of the secondary organ. These genes complement the initiation genes. Metastasis progression genes give advantages during the entire metastatic process by affecting general steps of tumor progression, such as tumor angiogenesis, inflammation, epithelial-to-mesenchymal transition or immune evasion. Importantly, metastasis progression genes may promote organotropic feature of metastasis by coupling molecular events controlling progression of primary tumor, tissuespecific spreading to distant organs and survival of metastatic cells $[35,50]$

Metastasis-associated signatures have been well characterized for lung and breast cancers. The expression of these genes in the primary tumor seems to predict organ-specific recurrence in patients $[17,59,60]$. An alternative interpretation of these observations, however, should be considered in light of the recent observation that in experimental tumors, metastatic cells can recirculate from the metastatic sites back to the primary tumor site by a mechanism of re-seeding [6]. Such a scenario is consistent with the existing correlation between primary tumor size, high proliferation rate and aggressive metastatic behavior leading to a poor prognosis [7]. If validated in human cancer, the ability of metastatic cells to colonize the primary tumor could explain the origin of "metastatic signatures" detectable in primary tumors and at the same time weaken the clinical predictive value of such signatures.

\section{Molecules mediating organ-specific metastasis}

In this paragraph we will briefly review molecular mechanisms of organ-specific metastasis recently identified by gene expression screens, gain- or loss-of-function experiments and pharmacological interventions. Although individual genes have been associated with organ-specific metastasis, they should be considered in a global context, as many of them act in concert (holistic view). Another complication is that one individual gene may be responsible for multiple effects while different genes may contribute to the acquisition of one particular feature. These pleiotropic and redundant effects exerted by a complex, dynamic and interactive network of multiple genes might explain the width and complexity of mechanisms underlying tissue-tropism of metastatic cells. This complexity and pleiotropism is reflected in the multiple, nonidentical, yet interchangeable prognostic signatures reported in breast cancer $[2,59,61]$. Initial evidence for the existence of genes driving organ-specific metastasis came from the identification of a 70-gene poor prognosis signature associated with the propensity to form distant metastases, through supervised clustering of a cohort of primary breast cancers (Rosetta-type poor prognosis signature) [62-65].

Subsequent experimental work mainly done by the Massagué's laboratory, described gene expression signatures associated with organ-specific metastasis in experimental models, which were validated in human breast cancer. Microarray-based comparison of the parental cancer cell lines to the organotropic metastatic 


\section{Primary tumor}

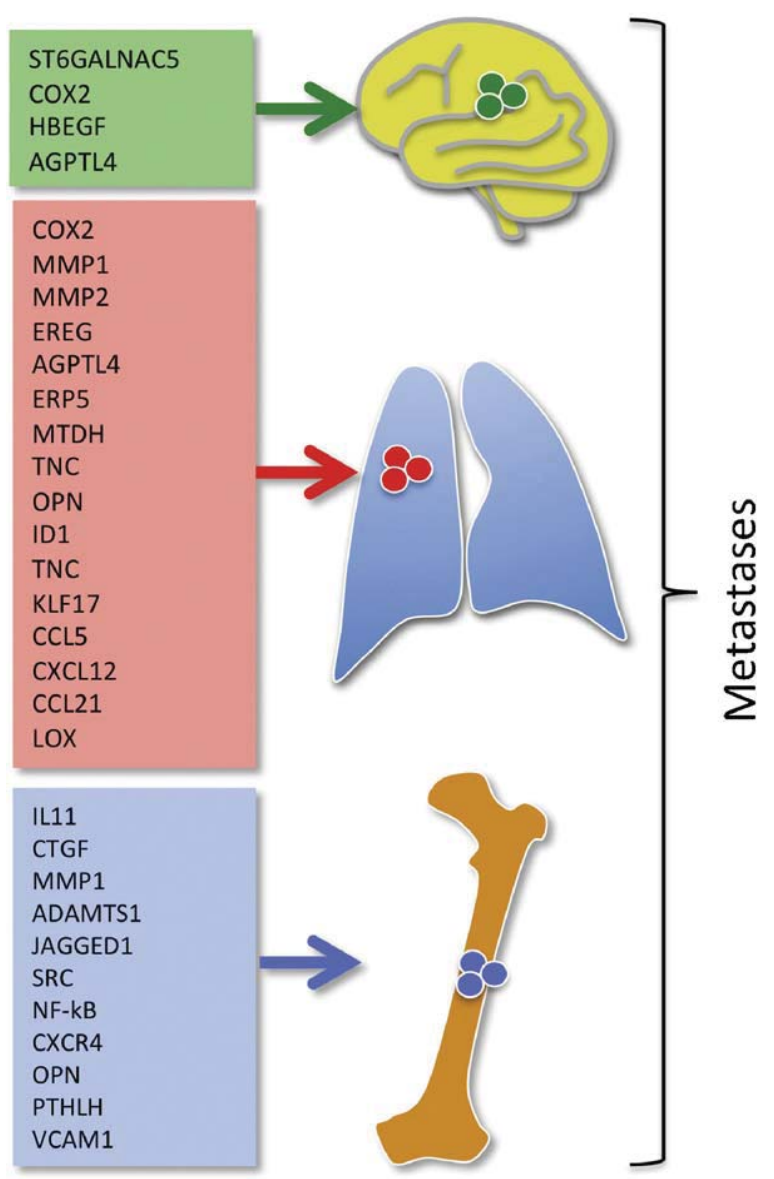

Fig. 2. Gene mediating organ specific breast cancer metastasis. Breast cancer genes promoting organ-specific metastasis to bone, lung and brain have been identified. They include proinflammatory molecules and chemokines/receptors (e.g. COX-, CXCL12/CXCR4), matrix-degrading and modifying enzymes (e.g. MMP1/2, LOX), adhesion and extracellular matrix molecules (e.g. VCAM-1, TNC, OPN), transcription factors (e.g. ID1, KLF17), intracellular signaling proteins (e.g. SRC, NF- $\kappa B$ ), cell communication proteins (JAGGED1, CTGF). Some genes promote seeding (e.g. ST6GALNAC5, AGPTL4), while others promote colonization (e.g. OPN, CXCR4). This list is meant to grow as novel genes are continuously reported. See Refs. $[33,48,53,74]$.

lines identified genes mediating metastasis to bone, lung and brain [53-55] (Fig. 2).

\subsection{Genes mediating metastasis to lungs and bones}

The bone metastasis signature revealed 43 over-expressed and 59 under-expressed genes in bone metastatic BoM1 cell line, isolated after one round of in vivo selection by intra-cardiac injection of the parental breast cancer cell line MDA-MB-231 [53]. Similarly, a lung metastasis signature consisting of 48 over-expressed and 47 under-expressed genes was identified from the lungmetastatic LM2 cell line, isolated after two rounds of in vivo selection by intra-vein injection of the same parental breast cancer cell line and recovery from the lung [54]. Among these genes only six were present in both signatures. Several lung metastasis genes were strongly associated with tumor invasiveness and aggressiveness, rather than functions in lung tissue, while bone metastasis-promoting genes were more related to bone microenvironment [58]. Bone metastatic cells take advantage from the physiological cycle of bone remodeling, mediated by genes such as IL-11, PTHrP and OPN, to survive and grow within the bone microenvironment.

\subsection{Genes mediating metastasis to the brain}

Using a similar approach, the same group further described a brain metastasis signature. In this model, breast cancer cells were injected directly into the arterial circulation, and then isolated from the brain. After two rounds of in vivo selection a brain metastatic line (BrM2) was obtained. The gene expression profile of the brain metastatic line identified 243 genes that were further analyzed for their association with brain relapse in independent clinical breast cancer data sets. The resulting 17-gene brain metastasis signature clinically correlated with basal-type ER-negative breast cancer subtype. One-third of the brain metastasis signature genes overlapped with genes of the lung metastasis signature. While these common genes cannot explain the specific and unique brain metastatic tropism they reveal that the brain metastasis signature is very different from the bone metastasis signature. The ST6GALNAC5 gene was found to be exclusively expressed in the brain-metastatic line and up-regulated in human brain metastasis samples. Functional validation experiments demonstrated a role in promoting brain metastasis by facilitating passage through the blood-brain barrier [55]. The significant overlap found between the lung and brain, but not bone metastasis signatures might be due to the functional similarity of the vasculature in lungs and brain. While the microvasculature in bone and liver is fenestrated, the one in lungs and brain is constituted by a continuous layer of endothelial cells with welldeveloped tight junctions. Therefore lung and brain metastatic cells need to overcome thigh vascular barriers to colonize their target organs. This notion is supported by the observation that some of the overlapping genes, such as COX2, ANGPTL4, LTBP1 and EGFRligands are functionally involved in controlling endothelial cell adhesion and vascular permeability.

\subsection{Clinical significance and limitations}

These signatures displayed an additional clinical value when applied to the 70-gene Rosetta-type poor prognosis signature and several independent cohorts of breast cancer patients. They appear to be robust, since the clinical correlation of metastasis-associated genes with the metastatic outcome and tropism was consistent across different patient cohorts analyzed and different microarray platforms $[10,50,61]$. From a mechanistic perspective, these signatures already contributed to make important advances in the characterization of novel molecular mediators of organ-specific metastasis. By coupling functional genomic approaches with more appropriate clinically-relevant metastasis models, it will be possible in the future to further improve our knowledge of metastasis organotropism with the aim of translating this knowledge into an improved clinical management of breast cancer metastasis [57]. It is important to keep in mind, however, that these signatures were obtained using experimental metastasis assays by directly inoculating cells into the circulation (i.e. intra-cardiac injections for bone and brain and lateral tail vein injections for lung metastases), thereby mimicking only the latter steps of metastatic process. Interestingly when these metastatic cell populations were orthotopically implanted in mouse mammary gland, the bone metastatic line grew with a comparable rate as the parental cell line, while the lung metastatic line showed a more rapid growth. This observation indicates that some organ-specific metastatic genes have already effects on primary tumor growth, supporting the notion that genes promoting primary tumor growth may also be relevant to metastasis formation. Future models should try to mimic spontaneous metastasis formation from primary tumors (see below). 


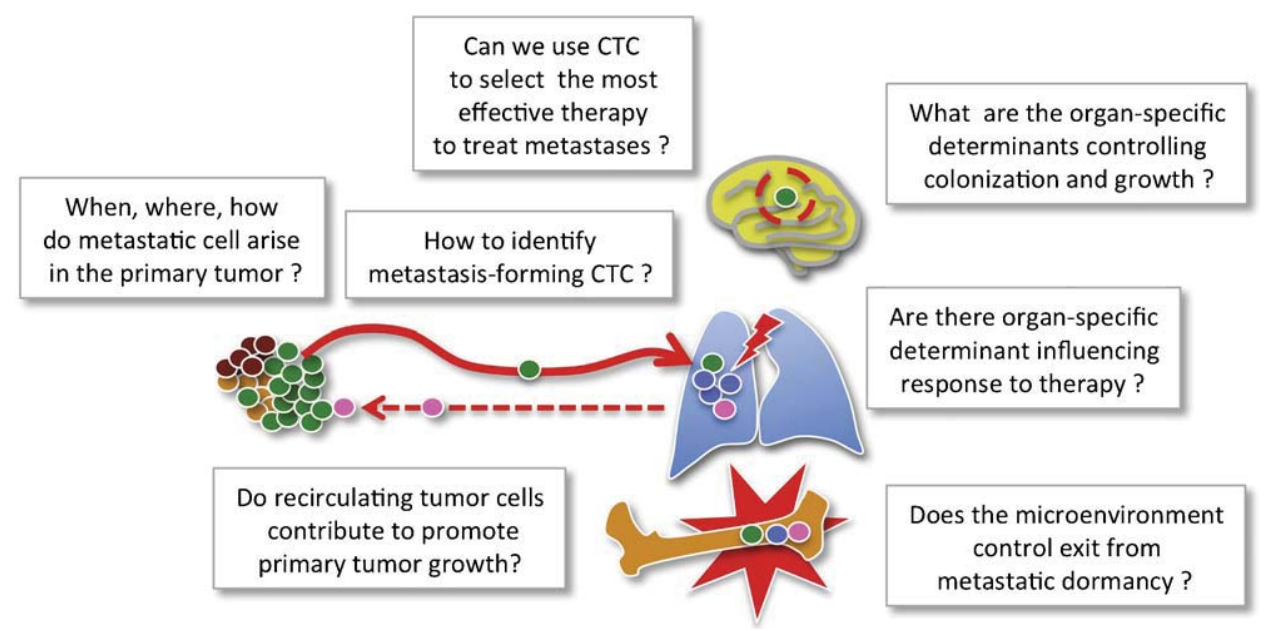

Fig. 3. Outstanding questions in breast cancer metastasis research. Innovative research approaches have provided important advances towards the identification of mechanisms of metastasis and associated molecules. A number of old questions have remained, however, and some new ones are emerging. Answering these questions might give some explanations why current therapies have limited impact on controlling metastatic disease and might open new avenues in designing more effective therapeutic approaches.

\section{Metastatic dormancy}

The observation that the vast majority of the metastases become evident only after removal or treatment of the primary tumor, indicates that disseminated cancer cells must be able to remain viable and silent for a long period of time, a phenomenon referred to as tumor dormancy, although "metastatic dormancy" would be more appropriate $[66,67]$. For example, metastases of ER-positive breast cancer, of prostate cancer or melanoma might become clinically apparent only years or decades after the surgical resection of even small primary lesions. The fact that relapses occur so late implies that these tumor cells are not fully capable to give rise to a secondary tumor at the moment of their seeding, yet they remain viable and progressively acquire this capacity through additional genetic and epigenetic modifications or cues from the tumor microenvironment $[14,68]$. This state of dormancy suggests that disseminated tumor cells might have low proliferative and low metabolic activities. This could represent a major obstacle limiting the efficiency of adjuvant chemotherapies, which are rather aimed at targeting highly active and proliferating cells. This might be the reason why adjuvant treatments often fail to eradicate dormant metastases [67,69]. However, prolonged dormancy is not seen in all cancer. For example, rapidly growing cancers, such as lung carcinoma and pancreatic adenocarcinoma display a quick metastatic evolution in secondary organs. These tumors rapidly acquire and develop genetic programs that confer colonization competence over a short period of time (weeks to months) following treatment of the primary tumor. Other cancers show mixed behaviors. For example, colorectal cancer might take decades for the transition from hyperplasia to dysplasia (adenoma), to in situ carcinoma and to invasive carcinoma, while it can rapidly progress to form metastases upon initiation of invasion $[67,70]$. This variability in the kinetic of metastatic progression is likely to reflect the great heterogeneity and complexity of the cellular and genetic events controlling metastasis among the different tumor types. Understanding metastatic dormancy is likely to open the way to novel approaches to the long-term control of cancer progression.

\section{Concluding remarks and outlooks}

Despite the successful therapeutic management of primary breast cancer, a significant fraction of the patients develop metastases, sooner or later. Breast cancer relapse is associated with a high rate of mortality. Since in patients the early steps in the metastasis process cannot be effectively targeted (as they have already occurred when metastases are detected), the latter steps of colonization and outgrowth at distant sites appear most suitable for therapeutic interventions. The development of strategies to effectively treat metastasis will benefit from the mechanistic understanding of metastasis organotropism as the results from the crosstalk between intrinsic properties of breast carcinoma cells and specific features of the colonized organ microenvironment. The recent reports that tenascin-C [71] and periostin [47] promote breast cancer stem cell survival in the lung and that VCAM-1 stimulates breast cancer cells survival and outgrowth in the lung and bone are important steps in this direction $[48,72]$.

\subsection{New models}

The experimental models of metastasis obtained by xenografting human breast cancer metastatic cell lines into immune-compromised mice through direct inoculation into the bloodstream emerged as valuable tools that allowed the identification of organ-specific metastatic signatures. However, these models are limited, since they only recapitulate the latter steps of a long, multistep process and bypass the need of a primary tumor. In view of the priming role of the primary tumor in metastasis formation (i.e. pre-metastatic niche model) and of the species-specificity of several cytokines involved in the cross-talk between cancer cells and microenvironment, it is therefore crucial to develop more clinically relevant models or spontaneous organ specific metastasis in immune-competent mice, based on orthotopic tumor cell implantation or spontaneous (transgenic) tumor formation. This will be relevant to the preclinical validation of candidate therapeutic targets. Orthotopic and spontaneous models of metastasis are expected to better fulfill the criteria necessary for developing anti-metastatic treatments eventually effective in patients, by disclosing failures or adverse side effects before reaching clinical testing [73]. We have recently developed an orthotopic and syngeneic model of breast cancer metastasis to the brain that allowed the identification of previously unsuspected genes conferring brain metastatic tropism to breast cancer cells (G. Lorusso, in preparation). Furthermore, it will be necessary to validate target genes identified in experimental models by analyzing their expression in public databases through bioinformatic 
approaches, as well as by direct validation of target genes on histological sections of clinical samples, early on during the discovery process [65]. The identification of patient subgroups co-expressing sets of genes that predispose to metastasis might eventually have a prognostic and predictive therapeutic value in the selection of the appropriate patient population to include in clinical trials of novel anti-metastatic agents.

\subsection{More questions}

Recently, innovative approaches, such the whole-genome sequencing of primary human tumors and matched metastases, coupled with proteomic-based approaches, have provided important advances in the identification of the molecular drivers of metastasis [57]. These approaches, together with relevant experimental models of disease progression, will contribute to improve our understanding of mechanisms of breast cancer metastasis. In spite of these advances, some outstanding questions in the field have still no answers. They include (Fig. 3): When, where, and how do metastatic cells arise within the primary tumor? Can we identify metastasis-forming circulating tumor cells among the whole population or circulating tumor cells and thereby estimate the risk of progression? Can we use circulating tumor cells to select the most effective therapy to prevent or treat metastases? What are the critical organ-specific determinants controlling colonization and growth, and can we impinge on them for therapeutic purposes? Are there organ-specific cues influencing response to therapy? Does the microenvironment control initiation and termination of metastatic dormancy? Do recirculating tumor cells contribute to promote primary tumor growth (and the bad prognostic signatures)? Answering these questions will by key to understand why current therapies have such a limited impact on controlling metastatic disease and will be instrumental in designing more effective drugs and therapeutic approaches.

\section{Conflict of interest statement}

The authors declare that there are no conflicts of interest.

\section{Acknowledgments}

Work in our laboratory is supported by the European Union under the auspices of the FP7 collaborative project TuMIC, contract no. HEALTH-F2-2008-201662; the Molecular Oncology Program of the National Center of Competence in Research (NCCR), a research instrument of the Swiss National Science Foundation; a collaborative research project (CCRP) of Oncosuisse (OCS-01812-12-2005), research grants from the Swiss National Science Foundation, the MEDIC Foundation and the ISREC Foundation. We apologize to those colleagues whose work could not be cited due to space limitations.

\section{References}

[1] Rabbani SA, Mazar AP. Evaluating distant metastases in breast cancer: from biology to outcomes. Cancer and Metastasis Reviews 2007;26(3-4):663-74.

[2] Valastyan S, Weinberg RA. Tumor metastasis: molecular insights and evolving paradigms. Cell 2011;147(2):275-92.

[3] Ding L, Ellis MJ, Li S, Larson DE, Chen K, Wallis JW, et al. Genome remodelling in a basal-like breast cancer metastasis and xenograft. Nature 2010;464(7291):999-1005.

[4] Yang X, Mortenson ED, Fu YX. Targeting and utilizing primary tumors as live vaccines: changing strategies. Cellular \& Molecular Immunology 2012;9(1):20-6.

[5] Steeg PS. Tumor metastasis: mechanistic insights and clinical challenges. Nature Medicine 2006;12(8):895-904.

[6] Kim MY, Oskarsson T, Acharyya S, Nguyen DX, Zhang XH, Norton L. Tumor self-seeding by circulating cancer cells. Cell 2009;139(7):1315-26.
[7] Comen E, Norton L, Massague J. Clinical implications of cancer self-seeding. Nature Reviews Clinical Oncology 2011;8(6):369-77.

[8] Lu X, Kang Y. Organotropism of breast cancer metastasis. Journal of Mammary Gland Biology and Neoplasia 2007;12(2-3):153-62.

[9] Minn AJ, Kang Y, Serganova I, Gupta GP, Giri DD, Doubrovin M. Distinct organspecific metastatic potential of individual breast cancer cells and primary tumors. The Journal of Clinical Investigation 2005;115(1):44-55.

[10] Nguyen DX, Bos PD, Massague J. Metastasis from dissemination to organspecific colonization. Nature Reviews Cancer 2009;9(4):274-84.

[11] Chiang AC, Massague J. Molecular basis of metastasis. The New England Journal of Medicine 2008;359(26):2814-23.

[12] Chaffer CL, Weinberg RA. A perspective on cancer cell metastasis. Science 2011;331(6024):1559-64.

[13] Shibue T, Weinberg RA. Metastatic colonization: settlement, adaptation and propagation of tumor cells in a foreign tissue environment. Seminars in Cancer Biology 2011;21(2):99-106.

[14] Lorusso G, Ruegg C. The tumor microenvironment and its contribution to tumor evolution toward metastasis. Histochemistry and Cell Biology 2008;130(6):1091-103.

[15] Luzzi KJ, MacDonald IC, Schmidt EE, Kerkvliet N, Morris VL, Chambers AF, et al. Multistep nature of metastatic inefficiency: dormancy of solitary cells after successful extravasation and limited survival of early micrometastases. American Journal of Pathology 1998;153(3):865-73.

[16] Husemann Y, Geigl JB, Schubert F, Musiani P, Meyer M, Burghart E, et al. Systemic spread is an early step in breast cancer. Cancer Cell 2008;13(1): 58-68.

[17] Ramaswamy S, Ross KN, Lander ES, Golub TR. A molecular signature of metastasis in primary solid tumors. Nature Genetics 2003;33(1):49-54.

[18] Albini A, Mirisola V, Pfeffer U. Metastasis signatures: genes regulating tumor-microenvironment interactions predict metastatic behavior. Cancer and Metastasis Reviews 2008;27(1):75-83.

[19] Koscielny S, Tubiana M, Le MG, Valleron AJ, Mouriesse H, Contesso G, et al. Breast cancer: relationship between the size of the primary tumour and the probability of metastatic dissemination. British Journal of Cancer 1984;49(6):709-15.

[20] Klein CA. Parallel progression of primary tumours and metastases. Nature Reviews Cancer 2009;9(4):302-12.

[21] Schmidt-Kittler O, Ragg T, Daskalakis A, Granzow M, Ahr A, Blankenstein TJ, et al. From latent disseminated cells to overt metastasis: genetic analysis of systemic breast cancer progression. Proceedings of the National Academy of Sciences of the United States of America 2003;100(13):7737-42.

[22] Braun S, Vogl FD, Naume B, Janni W, Osborne MP, Coombes RC, et al. A pooled analysis of bone marrow micrometastasis in breast cancer. The New England Journal of Medicine 2005;353(8):793-802.

[23] Slade MJ, Coombes RC. The clinical significance of disseminated tumor cells in breast cancer. Nature Clinical Practice Oncology 2007;4(1):30-41.

[24] Pantel K, Brakenhoff RH, Brandt B. Detection, clinical relevance and specific biological properties of disseminating tumour cells. Nature Reviews Cancer 2008;8(5):329-40.

[25] Hanahan D, Weinberg RA. Hallmarks of cancer: the next generation. Cell 2011;144(5):646-74.

[26] Pantel K, Brakenhoff RH. Dissecting the metastatic cascade. Nature Reviews Cancer 2004;4(6):448-56.

[27] Laubli H, Borsig L. Selectins promote tumor metastasis. Seminars in Cancer Biology 2010;20(3):169-77.

[28] Bockhorn M, Jain RK, Munn LL. Active versus passive mechanisms in metastasis: do cancer cells crawl into vessels, or are they pushed? The Lancet Oncology 2007;8(5):444-8.

[29] Padua D, Zhang XH, Wang Q, Nadal C, Gerald WL, Gomis RR, et al. Tgfbeta primes breast tumors for lung metastasis seeding through angiopoietin-like 4 . Cell 2008;133(1):66-77.

[30] Muller A, Homey B, Soto H, Ge N, Catron D, Buchanan ME, et al. Involvement of chemokine receptors in breast cancer metastasis. Nature 2001;410(6824):50-6.

[31] Wang J, Loberg R, Taichman RS. The pivotal role of cxcl12 (sdf-1)/cxcr4 axis in bone metastasis. Cancer and Metastasis Reviews 2006;25(4):573-87.

[32] Chambers AF, Groom AC, MacDonald IC. Dissemination and growth of cancer cells in metastatic sites. Nature Reviews Cancer 2002;2(8):563-72.

[33] Gupta GP, Massague J. Cancer metastasis: building a framework. Cell 2006;127(4):679-95.

[34] Gupta GP, Minn AJ, Kang Y, Siegel PM, Serganova I, Cordon-Cardo C, et al. Identifying site-specific metastasis genes and functions. Cold Spring Harbor Symposia on Quantitative Biology 2005;70:149-58.

[35] Nguyen DX, Massague J. Genetic determinants of cancer metastasis. Nature Reviews Genetics 2007;8(5):341-52.

[36] Wels J, Kaplan RN, Rafii S, Lyden D. Migratory neighbors and distant invaders: tumor-associated niche cells. Genes \& Development 2008;22(5):559-74.

[37] Psaila B, Lyden D. The metastatic niche: adapting the foreign soil. Nature Reviews Cancer 2009;9(4):285-93.

[38] Kaplan RN, Riba RD, Zacharoulis S, Bramley AH, Vincent L, Costa C, et al. Vegfr1positive haematopoietic bone marrow progenitors initiate the pre-metastatic niche. Nature 2005;438(7069):820-7.

[39] Kaplan RN, Psaila B, Lyden D. Bone marrow cells in the 'pre-metastatic niche': within bone and beyond. Cancer and Metastasis Reviews 2006;25(4):521-9.

[40] Thiery JP, Acloque H, Huang RY, Nieto MA. Epithelial-mesenchymal transitions in development and disease. Cell 2009;139(5):871-90. 
[41] Hiratsuka S, Nakamura K, Iwai S, Murakami M, Itoh T, Kijima H, et al. Mmp9 induction by vascular endothelial growth factor receptor-1 is involved in lungspecific metastasis. Cancer Cell 2002;2(4):289-300.

[42] Hiratsuka S, Watanabe A, Aburatani H, Maru Y. Tumour-mediated upregulation of chemoattractants and recruitment of myeloid cells predetermines lung metastasis. Nature Cell Biology 2006;8(12):1369-75.

[43] Rafii S, Lyden D. S100 chemokines mediate bookmarking of premetastatic niches. Nature Cell Biology 2006;8(12):1321-3.

[44] Erler JT, Bennewith KL, Nicolau M, Dornhofer N, Kong C, Le QT, et al. Lysyl oxidase is essential for hypoxia-induced metastasis. Nature 2006:440(7088):1222-6.

[45] Erler JT, Bennewith KL, Cox TR, Lang G, Bird D, Koong A, et al. Hypoxia-induced lysyl oxidase is a critical mediator of bone marrow cell recruitment to form the premetastatic niche. Cancer Cell 2009;15(1):35-44.

46] McAllister SS, Gifford AM, Greiner AL, Kelleher SP, Saelzler MP, Ince TA, et al. Systemic endocrine instigation of indolent tumor growth requires osteopontin. Cell 2008;133(6):994-1005.

[47] Malanchi I, Santamaria-Martinez A, Susanto E, Peng H, Lehr HA, Delaloye JF, et al. Interactions between cancer stem cells and their niche govern metastatic colonization. Nature 2011;481:85-9.

[48] Oskarsson T, Acharyya S, Zhang XH, Vanharanta S, Tavazoie SF, Morris PG, et al. Breast cancer cells produce tenascin c as a metastatic niche component to colonize the lungs. Nature Medicine 2011;17(7):867-74.

[49] Peinado H, Lavotshkin S, Lyden D. The secreted factors responsible for premetastatic niche formation: old sayings and new thoughts. Seminars in Cancer Biology 2011;21(2):139-46.

[50] Sethi N, Kang Y. Unravelling the complexity of metastasis - molecular understanding and targeted therapies. Nature Reviews Cancer 2011;11(10): $735-48$

[51] Paget S. The distribution of secondary growths in cancer of the breast. 1889 Cancer and Metastasis Reviews 1989;8(2):98-101.

[52] Fidler IJ. Selection of successive tumour lines for metastasis. Nature: New Biology 1973;242(118):148-9.

[53] Kang Y, Siegel PM, Shu W, Drobnjak M, Kakonen SM, Cordon-Cardo C, et al. A multigenic program mediating breast cancer metastasis to bone. Cancer Cell 2003;3(6):537-49.

[54] Minn AJ, Gupta GP, Siegel PM, Bos PD, Shu W, Giri DD, et al. Genes that mediate breast cancer metastasis to lung. Nature 2005;436(7050):518-24.

[55] Bos PD, Zhang XH, Nadal C, Shu W, Gomis RR, Nguyen DX, et al. Genes that mediate breast cancer metastasis to the brain. Nature 2009;459(7249): 1005-9.

[56] Yachida S, Jones S, Bozic I, Antal T, Leary R, Fu B, et al. Distant metastasis occurs late during the genetic evolution of pancreatic cancer. Nature 2010:467(7319):1114-7.

[57] Blanco MA, Kang Y. Signaling pathways in breast cancer metastasis - nove insights from functional genomics. Breast Cancer Research 2011;13(2):206.
[58] Lu X, Kang Y. Efficient acquisition of dual metastasis organotropism to bone and lung through stable spontaneous fusion between mda-mb-231 variants. Proceedings of the National Academy of Sciences of the United States of America 2009; 106(23):9385-90.

[59] Nevins JR, Potti A. Mining gene expression profiles: expression signatures as cancer phenotypes. Nature Reviews Genetics 2007;8(8):601-9.

[60] Hunter K, Welch DR, Liu ET. Genetic background is an important determinant of metastatic potential. Nature Genetics 2003;34(1):23-4 [author reply 5].

[61] Sotiriou C, Piccart MJ. Taking gene-expression profiling to the clinic: when will molecular signatures become relevant to patient care? Nature Reviews Cancer 2007;7(7):545-53.

[62] van't Veer LJ, Dai H, van de Vijver MJ, He YD, Hart AA, Mao M, et al Gene expression profiling predicts clinical outcome of breast cancer. Nature 2002;415(6871):530-6.

[63] van de Vijver MJ, He YD, van't Veer LJ, Dai H, Hart AA, Voskuil DW, et al. A gene-expression signature as a predictor of survival in breast cancer. The New England Journal of Medicine 2002;347(25):1999-2009.

[64] Buyse M, Loi S, van't Veer L, Viale G, Delorenzi M, Glas AM, et al. Validation and clinical utility of a 70-gene prognostic signature for women with nodenegative breast cancer. Journal of the National Cancer Institute 2006;98(17): 1183-92.

[65] Sotiriou C, Pusztai L. Gene-expression signatures in breast cancer. The New England Journal of Medicine 2009;360(8):790-800.

[66] Naumov GN, MacDonald IC, Chambers AF, Groom AC. Solitary cancer cells as a possible source of tumour dormancy? Seminars in Cancer Biology 2001;11(4):271-6.

[67] Goss PE, Chambers AF. Does tumour dormancy offer a therapeutic target? Nature Reviews Cancer 2010;10(12):871-7.

[68] Joyce JA, Pollard JW. Microenvironmental regulation of metastasis. Nature Reviews Cancer 2009;9(4):239-52.

[69] Aguirre-Ghiso JA. Models, mechanisms and clinical evidence for cancer dormancy. Nature Reviews Cancer 2007;7(11):834-46.

[70] Steeg PS, Theodorescu D. Metastasis: a therapeutic target for cancer. Nature Clinical Practice Oncology 2008;5(4):206-19.

[71] Chen $\mathrm{Q}$ Zhang XH, Massague J. Macrophage binding to receptor vcam-1 transmits survival signals in breast cancer cells that invade the lungs. Cancer Cell 2011;20(4):538-49.

[72] Lu X, Mu E, Wei Y, Riethdorf S, Yang Q, Yuan M, et al. Vcam-1 promotes osteolytic expansion of indolent bone micrometastasis of breast cancer by engaging alpha4beta1-positive osteoclast progenitors. Cancer Cell 2011;20(6):701-14.

[73] Francia G, Cruz-Munoz W, Man S, Xu P, Kerbel RS. Mouse models of advanced spontaneous metastasis for experimental therapeutics. Nature Reviews Cancer $2011 ; 11(2): 135-41$.

[74] Gupta GP, Nguyen DX, Chiang AC, Bos PD, Kim JY, Nadal C, et al. Mediators of vascular remodelling co-opted for sequential steps in lung metastasis. Nature 2007;446(7137):765-70 